\title{
Pattern of Breastfeeding and its impact on Post-Partum Amenorrhoea: A cross-sectional study
}

\section{G Singh}

\begin{abstract}
Citation
G Singh. Pattern of Breastfeeding and its impact on Post-Partum Amenorrhoea: A cross-sectional study. The Internet Journal of Epidemiology. 2006 Volume 4 Number 1.
\end{abstract}

DOI: $\underline{10.5580 / \mathrm{e} 19}$

\begin{abstract}
There is a known relationship between period of breastfeeding and period of post-partum amenorrhoea (PPA). The objective of this paper was to determine the relationship between pattern of breastfeeding like full or partial, at fixed time or on demand of child, with the length of PPA in a cross-sectional study on 112 women. Median duration of PPA was observed as 5 months. Subjects were classified as PPA $\leq 5$ months and PPA $>5$ months. Odds ratio and its $95 \% \mathrm{Cl}$ for both full/partial and fixedtime/on-demand pattern of breastfeeding when determined resulted not statistically significant.
\end{abstract}

\section{INTRODUCTION}

Breastfeeding besides providing required nutrients for proper growth and development of a child ensures in developing immunity to protect from infections thereby promoting child survival and to mother gives some protection against early conception by delayed resumption of ovulation i.e. by increasing the period of post-partum amenorrhoea (PPA). PPA period in a woman is an interval that begins at the termination of a conception and ends with the resumption of ovulation. It has been recommended that full breastfeeding should be continued upto six months thereafter adequate complementary items should be introduced alongwith breastfeeding. $\left[_{1}\right]$ It has been reported that PPA period is positively correlated with period and practice of breastfeeding though, the length of PPA in women may also vary depending on a number of factors. $\left[{ }_{2}, 3,4\right]$

The aim of this study was to determine the relationship between practice of breastfeeding like full or partial, at fixed time or on demand of child, with the period of PPA in a cross-sectional study of 112 women.

The study reflected some interesting findings, which may be taken as lead to further examine the phenomenon based on a large sample incorporating other important factors also.

\section{METHOD}

In a cross-sectional study a sample of 112 women was selected from child immunization centre at Varanasi, India in the year 2004 where they came with their youngest child for vaccination. Women were interviewed through a study proforma. The data on duration of breastfeeding, full and partial breastfeeding, feeding at fixed time or on demand of the child was collected. The duration of PPA in months was also recorded. The age range of women under study was 20-34 years. Women were selected regardless of parity. A large proportion of women were housewives belonging to middle class family and coming from urban area. Women were excluded if taken any hormonal treatment after last delivery to prolong the time of resumption of ovulation to ensure prevention of early conception, and if were not sure about time of their first menstruation after last delivery.

\section{RESULTS}

The analysis of data revealed some interesting results as shown in Table-1and 2.

As the median time of PPA for the observed data was found 5 months therefore, subjects were divided into two categories one with PPA $\leq 5$ months and other with PPA $>5$ months. It was observed that among women with PPA $>5$ months $(n=20), 80 \%$ practiced full breastfeeding and $20 \%$ partial breastfeeding whereas, for this group the percentage feeding at fixed time was equal to the percentage feeding on demand of the child. So the role of full breastfeeding practice in enhancing PPA was noticed but the time of feeding showed no difference. 
Table-1 shows distribution of subjects according to full/partial breastfeeding and PPA period. $61.6 \%$ reported full breastfeeding while $38.4 \%$ partial breastfeeding. Odds ratio $(\mathrm{OR})$ with $95 \%$ confidence interval $(\mathrm{CI})$ and its statistical significance were determined. Odds refers to ratio of occurrence to non-occurrence of event. So here in Table-1we have 53/16 = 3.31 showing that for full breastfeeding women there will be 331 women with PPA $\leq 5$ months per 100 women with PPA $>5$ months. Whereas, $39 / 4=9.75$ shows that for partial breastfeeding women there will be 975 women with PPA $\leq 5$ months per 100 women with PPA $>5$ months. So in partial breastfeeding chances of PPA $>5$ months is smaller as compared to full breastfeeding. In other words, in partial breastfeeding chances of PPA $\leq 5$ months is higher compared to full breastfeeding women. However, this observation may not be generalized as the value of $\mathrm{OR}=0.3397$ (95\% CI as 0.1053 to 1.0959 ) was found not statistically significant.

Table-2 shows distribution of subjects according to fixedtime/on-demand breastfeeding and PPA period. $56.3 \%$ reported breastfeeding at fixed time while $43.8 \%$ breastfeeding on demand of child. Odds ratio $(\mathrm{OR}=1.3589)$ with $95 \%$ CI (0.5156 to 3.5819) and its statistical significance were also determined as above. Similar to Table- 1 here also the difference was found not statistically significant.

Thus data could not provide sufficient evidence to conclude that full/partial and fixed-time/on-demand practice of breastfeeding may produce some difference in the period of PPA.

\section{Figure 1}

Table 1

\begin{tabular}{|l|lr|lr|rr|}
\hline $\begin{array}{l}\text { Practice of } \\
\text { Breastfeeding }\end{array}$ & \multicolumn{2}{|l|}{ PPA $\leq 5$ months } & \multicolumn{2}{|l|}{ PPA $>5$ months } & \multicolumn{2}{|l|}{ Total } \\
\hline Full breastfeeding & 53 & 76.8 & 16 & 23.2 & 69 & 61.6 \\
& & & & & & \\
\hline Partial breastfeeding & 39 & 90.7 & 04 & 09.3 & 43 & 38.4 \\
& & & & & & \\
\hline Total & 92 & 82.1 & 20 & 17.9 & 112 & 100.0 \\
\hline & & & & & \\
\hline
\end{tabular}

\section{Figure 2}

Table 2

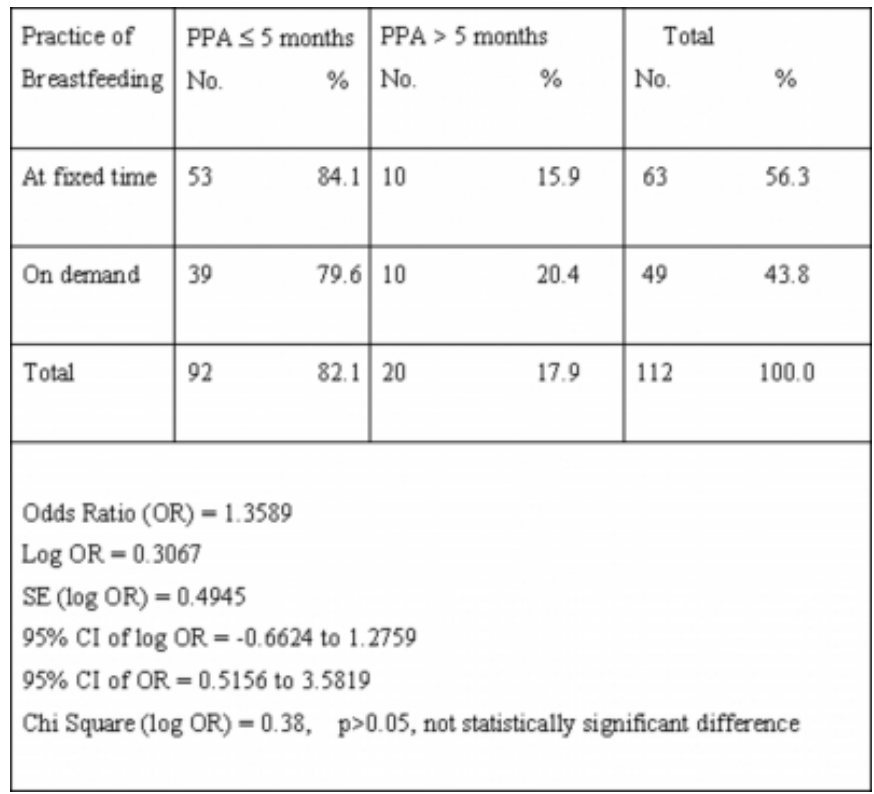

\section{DISCUSSION}

The duration and pattern of breastfeeding varies considerably among women depending on a number of biological and socio-cultural factors. Similar is the variation in case of post-partum amenorrhoea. A number of studies has found positive correlation between duration of breastfeeding and duration of post-partum amenorrhoea.[3,4] As regards pattern of breastfeeding like full or partial, at fixed time or on demand of the child etc. these may also play 
some role in lengthening or shortening the period of PPA. The present small cross-sectional study based on self reported data had observed some difference in duration of PPA depending on the pattern of breastfeeding though, the difference could not be found statistically significant. The threshold of 5 months PPA period taken for categorizing was based on the observed median PPA duration. An increase or decrease in this value in future studies when conducted may reveal similar or some other picture. This interesting finding may be taken as input for other researchers for further exploring the mechanism incorporating other aspects also.

\section{CORRESPONDENCE TO}

Dr.Girish Singh 6, Ganga Bagh Colony, Lanka Varanasi -
221005 India Phone: 91-542-2367304 e-mail:

drgirishsingh@yahoo.com

\section{References}

1. Boland M. Exclusive breastfeeding should continue to six months. Paediatrics and Child Health 2005; 10 (3): 148.

2. Zhang LY, Liu YR, Shah IH, et.al. Breastfeeding, amenorrhoea and contraceptive practice among post partum women in Zibo, China. Eur J Contracept Reprod Health Care 2000; 7(3): 121-6.

3. Singh G. Regression analysis of the effect of full and partial lactation on post-partum amenorrhoea in lower middle class Indian females. Indian Journal of Medical Sciences 1997; 51(6): 181-5.

4. Singh KK, Suchindran CM, Singh RS. Smoothed breastfeeding durations and waiting time to conception. Soc Biol 1994; 41(3-4): 229-39. 


\section{Author Information}

Girish Singh, Ph.D.

Biostatistics Unit, Department of Basic Principles, Institute of Medical Sciences, Banaras Hindu University 